03.13

\title{
Распределение интенсивности трехволновой дифракции от дислокационных эпитаксиальных слоев в обратном пространстве
}

\author{
(C) P.H. КютT \\ Физико-технический институт им. А.Ф. Иофффе РАН \\ Санкт-Петербург, Россия \\ E-mail: r.kyutt@mail.ioffe.ru
}

(Поступила в Редакцию 3 ноября 2017 г.)

\begin{abstract}
Экспериментально иссследована трехволновая дифракция рентгеновских лучей в сильнонарушенных эпитаксиальных слоях $\mathrm{GaN}$ и $\mathrm{ZnO}$. Построены карты распределения интенсивности в обратном пространстве в координатах $q_{\theta}$ и $q_{\varphi}$ для наиболее интенсивной трехволновой комбинации $(10 \overline{1} 0) /(\overline{1} 011)$ путем последовательных $\theta$ и $\varphi$-сканирования. Обнаружена нетривиальная форма $\theta$-сечений этих контуров вдали от $\varphi$-центра отражения, неодинаковая для разных образцов. Для $\theta$-кривых в центре отражения наблюдается единый пик, аппроксимируемый функцией Войта со степенным спадом интенсивности на крыльях, при этом закон спаданий $(-4.5--5.0)$ заметно больше, чем для аналогичнх кривых двухволновой дифракции, и не зависит от плотности и распределения дислокаций в слоях. В некоторых пленках имеет место крупноблочная структура, при этом из распределения в обратном пространстве следует, что эти блоки развернуты друг относительно друга вокруг нормали к поверхности, что заставляет предположить между ними малоугловые границы, состоящие исключительно из краевых дислокаций.
\end{abstract}

Работа выполненаа при частичной поддержке гранта РФФИ № 16-02-00702.

DOI: 10.21883/FTT.2018.04.45676.311

\section{1. Введение}

Многоволновая дифракция рентгеновских лучей, известная с 1937 г., редко используется для иссследований эпитаксиальных пленок и других поверхностных слоев. В основном, ее применение ограничивалось определением параметров решетки и деформаций в них [1-3]. Лишь в последние годы появились работы, касающиеся влияния структурных дефектов (в основном, дислокаций) на поведение картины трехволной дифракции, измеренной по схеме Реннингера [4-9]. Такая возможность появилась в связи с вводом в оборот сильнонарушенных эпитаксальных слоев III-нитридов и $\mathrm{ZnO}$, имеющих на современном этапе очень высокую плотность дислокаций (до $10^{10} \mathrm{~cm}^{-2}$ ), что дает хорошую возможность исследовать закономерности трехволновой дифракции в зависимости от структурного совершенства.

В работе [4] были измерены пики Реннингера от $\mathrm{GaN}$ и $\mathrm{ZnO}$ и показано соответствие угловых положений и относительных интенсивностей теоретическим расчетам. В [5] изучалось влияние буферного слоя AlN на реннингеровскую диаграмму от верхнего слоя GaN. B [6] были проанализированы трехволновые пики от $\mathrm{ZnO}$ в геометрии нескольких запрещенных первичных отражений.

В [7-9] была более подробно экспериментально исследована трехволновая дифракция в пленках $\mathrm{GaN}, \mathrm{AlN}$, $\mathrm{AlGaN}$ и $\mathrm{ZnO}$, выращенных на подложках сапфира и $\mathrm{SiC}$, разной толщины и разной степени структурного совершенства. В результате был выявлен ряд закономерностей зависимости дифракционной картины от дислокационной структуры, которые, к сожалению, еще не все получили соответствующее объяснение.

В [9] была впервые получена карта распределения интенсивности в обратном пространстве вокруг первичного узла 0001 для одного из трехволновых пиков Реннингера для эпитаксального слоя $\mathrm{GaN}$ с большой плотностью дислокаций. Правда, она была измерена по стандартной схеме $\theta$ и $\theta-2 \theta$ и мало чем отличалась (кроме ширины $\theta$-сечения) от обычных карт для симметричных брэгговских отражений.

В одной из работ E. Rosmanith [10] измерено и рассчитано $\theta-\varphi$ распределение интенсивности диаграммы Реннингера для кремния с первичным отражением 222, однако сделано это, как и в других работах, для мозаичного монокристалла со сферическими кристаллитами и во внимание принимаются только инструментальные факторы, речи о структурных нарушениях нет.

В настоящей работе распределение интенсивности измеряется как сочетание $\theta$ - и $\varphi$-мод сканирования с целью получения дополнительной информации о дефектной структуре.

\section{2. Методика}

Трехволновая дифракция возникает, когда наряду с узлами обратной решетки 0 и $H_{1}$ (случай стандартной двухволновой дифракции) на сферу Эвальда попадает еще один узел $H_{2}$. В этом случае брэгговские условия 
Сравнение параметров формы дифракционных пиков $\theta$-сканирования трехволновой и двухволновой дифракции

\begin{tabular}{|c|c|c|c|c|c|c|c|c|c|}
\hline \multirow{2}{*}{ № } & \multirow{2}{*}{ Образец } & \multicolumn{2}{|c|}{ FWHM, arcsec } & \multicolumn{2}{|c|}{$w(G), \operatorname{deg}$} & \multicolumn{2}{|c|}{$w(L), \operatorname{deg}$} & \multicolumn{2}{|c|}{ Наклон } \\
\hline & & 0002 & $10 \overline{1} 0 / \overline{1} 011$ & 0002 & $10 \overline{1} 0 / \overline{1} 011$ & {$[\mathrm{C} 0002$} & $10 \overline{1} 0 / \overline{1} 011$ & 0002 & $10 \overline{1} 0 / \overline{1} 011$ \\
\hline 1 & GaN-426 & 170 & 930 & 0.041 & 0.223 & 0.017 & 0.048 & -3.0 & -4.0 \\
\hline 2 & GaN-161 & 375 & 2600 & 0.096 & 0.53 & 0.015 & 0.34 & -3.5 & -5.0 \\
\hline 3 & GaN-72 & 793 & 3160 & 0.185 & 0.606 & 0.065 & 0.468 & -4.0 & -4.0 \\
\hline 4 & GaN-30 & 870 & 2480 & 0.020 & 0.555 & 0.042 & 0.26 & -4.5 & -4.1 \\
\hline 5 & $\mathrm{ZnO}$ & 95 & 1690 & 0.017 & 0.53 & 0.017 & 0.34 & -2.2 & -5.0 \\
\hline
\end{tabular}

будут соблюдаться не только для отражений $H_{1}$ и $H_{2}$ с векторами обратной решетки $0 \mathrm{H}_{1}$ и $0 \mathrm{H}_{2}$, но автоматически и для рефлекса с вектором $\mathrm{H}_{1} \mathrm{H}_{2}=0 \mathrm{H}_{1}-\mathrm{OH}_{2}$ (рис. 1). На рис. 1 треугольник $P 0 H_{1}$ определяет плоскость рассеяния первичного отражения, $\mathrm{POH}_{2}-$ вторичного отражения, а $\mathrm{PH}_{2} \mathrm{H}_{1}-$ связующего рефлекса, при этом для последнего $\mathbf{k}_{2}$ будет волновым вектором падающей волны, a $\mathbf{k}_{1}$ - отраженной. Трехволновые комбинации находятся путем вращения образца вокруг вектора $\mathbf{H}_{1}$ (в данном случае совпадающим с нормалью к поверхности). Такая схема измерений была предложена Максом фон Реннингером в 1937 г. и по праву называется схемой Ренингера.

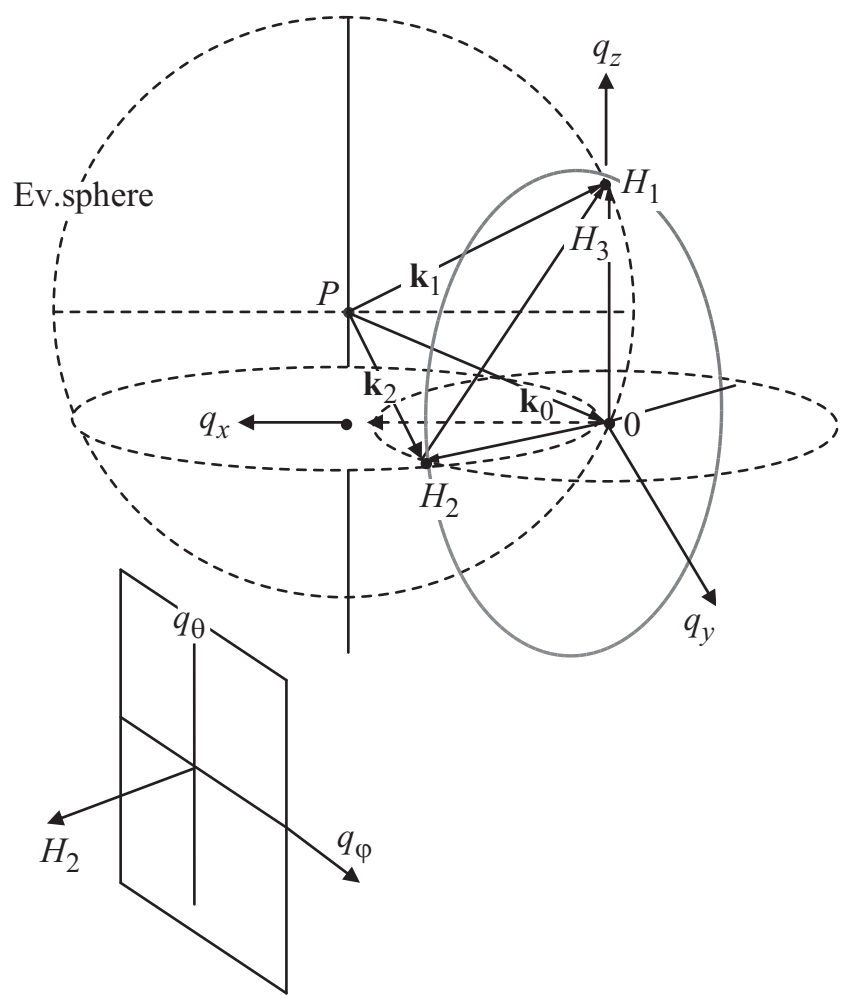

Рис. 1. Схема расположения узлов обратной решетки на сфере Эвальда в случае трехволновой дифракции и их смещение при $\theta$ - и $\varphi$-сканировании (вверху) и оси распределения интенсивности в обратном пространстве (внизу).
Объектами исследования были образцы с пленками $\mathrm{GaN}$ и $\mathrm{ZnO}$, выращенными на с-грани сапфира, разного структурного совершенства. Эти образцы были ранее детально изучены методами обычной двухволновой дифрактометрии. Перечень образцов и некоторые их свойства приведены в табл. 1.

Экспериментальные измерения проводились на рентгеновском дифрактометре в двухкристальном варианте на $\mathrm{Cu} K_{\alpha}$-излучении. Монохроматором служил монокристалл Ge в отражении 111. Первичная интенсивность (после монохроматора) достигала значения $I_{0}=4 \cdot 10^{6} \mathrm{imp} / \mathrm{s}$. Первичным было взято запрещенное для вюрцитных структур отражение 0001. Двумерные распределения в координатах $q_{\varphi}$ и $q_{\theta}$ (рис. 1) измерялись путем пошагового азимутального сканирования (вокруг нормали к поверхности образца) и сканирования по брэгговскому углу (поворот образца вокруг оси $\left.q_{y}\right)$.

Из трехволновых рефлексов была выбрана комбина-

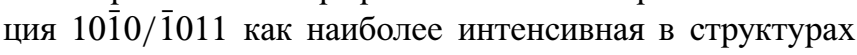
III-нитридов с вюрцитной решеткой. Кроме того, вторичное отражение $10 \overline{1} 0$ имеет вектор обратной решетки, перпендикулярный первичному вектору [0001] (т.е. это чистое Лауэ-отражение), что упрощает построение карты интенсивности в обратном пространстве.

\section{3. Результаты и их обсуждение}

Контуры распределения интенсивности для двух образцов приведены на рис. 2. Соответствующая картина должна представлять собой двумерное сечение узла обратной решетки вторичного рефлекса $H_{2}$ сферой Эвальда. Выявляются следующие свойства полученной дифракционной картины.

Во-первых, контуры имеют для всех структур симметричную форму с центром при одном и том же углах падения и отражения. Отсюда следует, что эти направления не зависят от азимутального угла $\varphi$ в пределах распределения данного рефлекса.

Во-вторых, обращает на себя внимание тройной контур в центре распределения, вытянутый в направлении $q_{\varphi}$ для нескольких образцов (пример на рис. 2,a). Такой вид обусловлен крупноблочной структурой пленки с разворотом блоков друг относительно друга вокруг 


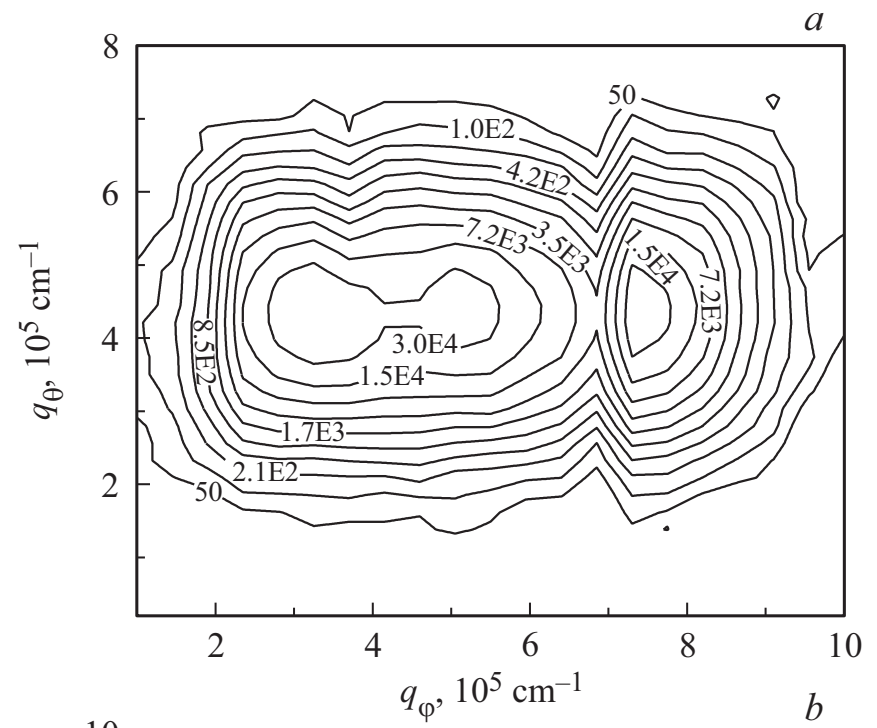

которого не меняется в зависимости от азимутального угла, что подтверждает вышеизложенный вывод.

Однако при удалении от центра $\theta$-кривые меняют свою форму. Для образцов 2 и 3 они расщепляются на три максимума (см. рис. 3,a). При этом центральный пик остается на месте, а боковые разъезжаются с увеличением угла $\varphi$, отсчитываемого от центра отражения. Именно такое строение поперечных сечений приводит к тому, что вдали от центра контуры равной интенсивно-
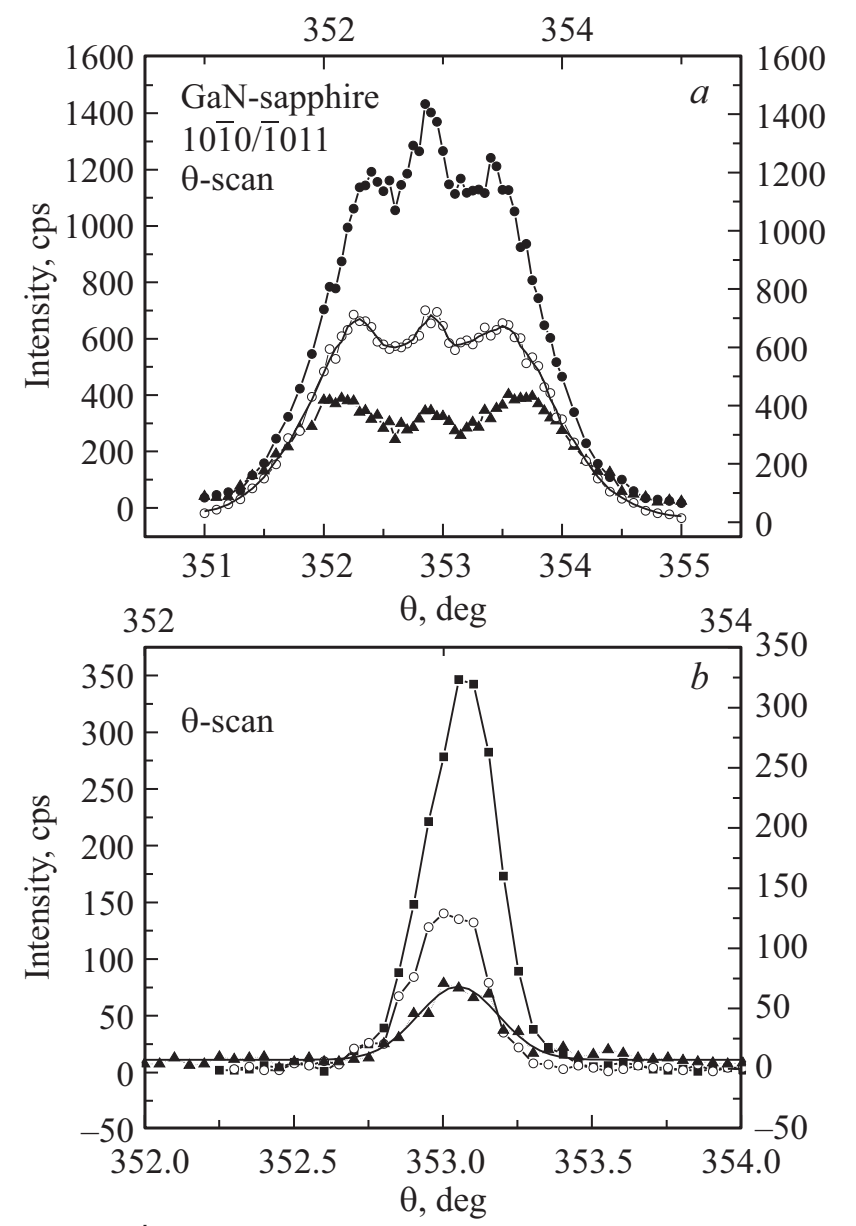

Pис. 2. Распределение интенсивности в обратном пространстве в плоскости, перпендикулярной вектору обратной решетки вторичного рефлекса $\left(q_{\theta}-q_{\varphi}\right)$ для образцов № $1(a)$ и № $3(b)$.

нормали к поверхности (см. об этом в [7]). При этом из рис. 2, $a$ видно, что центры рассеяния этих блоков находятся на одной линии $q_{\theta}$, что показывает, что эти блоки не развернуты вокруг осей, параллельных поверхности. Можно сказать, что границы между этими блоками образованы скоплениями краевых дислокаций (дислокационными стенками), поскольку присутствие винтовых компонент привело бы к разориентации блоков и изменению угла отражения.

Сечения контуров по линии $q_{\theta}(\theta$-кривые) позволяют более детально изучить особенности распределения интенсивности. В центральной области отражения они представляют собой единый пик, угловое положение

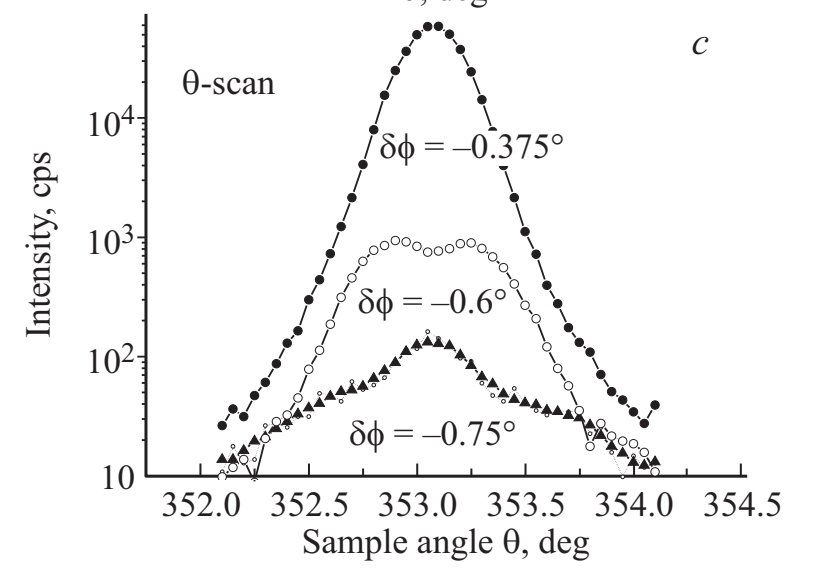

Рис. 3. Транформация кривых $\theta$-сканирования при изменении азимутального угла $\phi$ от центра отражения для образцов № $3(a)$, № 1 (b) и № $2(c)$. 
сти на картах распределения не имеют овальной (дискообразной) формы. Данная картина похожа на рассеяние от монокристаллов с дефектами кулоновского типа с когерентным и диффузным рассеянием. Однако в нашем случае большой плотности дислокаций о когерентном рассеянии речи быть не может. Другое объяснение состоит в том, что это так называемые „псевдопики“, имеющие чисто инструментальный характер. Такие пики характерны для сечений в двухволновой дифракции, где они вызваны конечной шириной отражения и хвостами отражений от монохроматора и анализатора (при трехкристальных измерениях).

В принципе существование „псевдопиков“ возможно и в трехволновой дифракции при $\theta-\varphi$-измерениях, и может быть связано с конечной вертикальной расходимостью как падающего, так и дифрагированного пучков. Однако такое объяснение противоречит другим экспериментальным фактам. А именно, для образца № 1 мы вовсе не наблюдаем боковых пиков, а только один центральный (рис. $3, b$ ), а для образца 2 нет центрального пика, а только боковые, которые сближаются при приближении (по углу $\varphi$ ) к центру отражения и затем сливаются в один (рис. 3,c). Аналогичный (как на рис. $3, c$ ) вид имеют и кривые от слоя $\mathrm{ZnO}$. (Заметим, что картина рассеяния измерялась для всех образцов при одних и тех же инструментальных условиях.)

При этом такое различие никак не связано ни с плотностью дислокаций, ни с их распеделением в эпитаксиальном слое. Так, к примеру, для пленок образцов № 1 ( $\mathrm{GaN})$ и № 5 ( $\mathrm{ZnO})$, показывающих наилучшее структурное совершенство с минимальной плотностью прорастающих винтовых дислокаций (по данным измерений двухволновой дифракции), поперечные сечения имеют совершеннно различный вид. В то же время для образцов 2 и 3, имеющих разный тип распределения дислокаций (в первом случае прямолинейные прорастающие, во втором - хаотическое распределение по объему - форма $\theta$-кривых вдали от центра и их зависимость от азимутального угла одинакова.

В центре азимутального отражения $\theta$-кривая отражения представляет собой одиночный пик симметричной формы. Его форма может быть аппроксимирована функцией Войта, но только в центральной части, а на крыльях преобладает степенной закон изменения интенсивности. В этом смысле никакого различия между двухволновыми пиками и трехволновыми нет.

В табл. 1 приведены сравнительные параметры $\theta$-пиков, измеренных в двухволновой (симметричное брэгговское отражение 0002) и трехволновой дирфкции (рефлекс $(10 \overline{1} 0 /(\overline{1} 011))$. Видно, что угловая ширина трехволновых пиков значительно больше, чем двухволновых, что было отмечено и раньше [9]. Это вызывает удивление тем, что при уходе первичного узла 0001 со сферы Эвальда интенсивность тройного рефлекса сохраняется.
Во-вторых, при аппроксимации функцией Войта, доля лоренцовской по сравннию с гауссовской составляющей в трехволновых пиках заметно выше, чем в двухволновых. Поскольку в первом случае задействованы не только винтовые, но и краевые дислокации, можно предположить большую степень корреляции между ними.

Падение интенсивности на хвостах кривых также отличается в двухволновом и трехволновом случаях. Если для первых степенной закон спадания неодинаков для разных образцов и зависит, в основном, от степени регулярности в распределении дислокаций [10], то во втором - примерно одинаков и интенсивность падает быстрее, чем в симметричной брэгговской геометрии. В принципе, это может быть объяснено тем, что мы имеем суммарное падение интенсивности как брэгговского, так и лауэвского рефлексов. Но такой вывод, вообще говоря, противоречит предыдущему утверждению.

\section{4. Заключение}

Распределение интенсивности в обратном пространстве в координатах $q_{\theta}$ и $q_{\varphi}$ в принципе дает форму узла обратной решетки вторичного отражения, которую впрямую не получить. При этом существует разница между $\varphi$ - и $\theta$-сканированием. В первом случае узел первичного отражения остается на своем месте. Сферу Эвальда пересекает только вторичное отражение. Во втором случае оба узла движутся относительно сферы Эвальда. Однако для симметричного брэгговского (первичного) отражения распределение в любом случае представляет собой диск, перпендикулярный $\mathbf{H} \| \mathbf{n}$. Не совсем ясна остается роль третьего освязующего отражения. По геометрии сферы Эвальда оно всегда должно оставаться в центре.

Надо отметить, что при такой плотности дислокаций (выше $10^{7} \mathrm{~cm}^{-2}$ ), очевидно, нет смысла говорить о первичной экстинкции - кинематическое приближение подразумевается само собой. Поскольку в данной работе речь не идет о сравнениии интенсивности различных отражений, то и вопросы влияния вторичной экстинкции остаются вне ее пределов. Конечно, вызывает уддивление форма сечений контуров вдали от центра. Чтобы прояснить этот вопрос, потребуются дальннейшие измерения с использованием других трехволновых комбинаций.

\section{Список литературы}

[1] J.M. Sasaki, L.P. Cardoso, C. Campos, K.J. Roberts, G.F. Clark, E. Pantos, M.A. Sacilott. J. Appl. Phys. 79, 3492 (1996).

[2] D. Korytar, C. Ferrary, Z. Bochnicek. J. Appl. Cryst. 31, 570 (1998). 
[3] R.O. Freitas, T.E. Lamas, A.A. Quivy, S.L. Morelhao. Phys. Status Solidi A 204, 2548 (2007).

[4] J. Blasing, A. Krost. Phys. Status Solidi A 201, 17 (2004).

[5] J. Blassing, A. Krost, J. Hertkorn. J. Appl. Phys. 105, 033504 (2009).

[6] M. Grundman, M. Scheibe, M. Loren, J. Blasing, A. Krost. Phys. Status Solidi A 251, 850 (2014).

[7] Р.Н. Кютт. Письма в ЖТФ 36, 15, 14 (2010).

[8] Р.Н. Кютт. ЖТФ 81, 5, 81 (2011).

[9] R.N. Kyutt, M.P. Scheglov. J. Appl. Cryst. 46, 861 (2013).

[10] Р.Н. Кютт. ЖТФ 87, 578 (2017).

[11] E. Rossmanith. J. Appl. Cryst. 33, 1405 (2000). 